\title{
FORCES ANALYSIS FOR MICROMANIPULATIONS IN DRY AND LIQUID MEDIA
}

\author{
Michaël Gauthier ${ }^{1 *}$, Stéphane Régnier ${ }^{2}$, Patrick Rougeot ${ }^{1}$ and Nicolas Chaillet $^{1}$ \\ ${ }^{1}$ Laboratoire d'Automatique de Besançon \\ CNRS - ENSMM - UFC \\ 24, rue Alain SAVARY \\ 25000 Besançon (France) \\ gauthier, rougeot, chaillet@ens2m.fr \\ ${ }^{2}$ Laboratoire de Robotique de Paris \\ CNRS - UPMC \\ BP 61 \\ 92265 Fontenay Aux Roses (France) \\ regnier@robot.jussieu.fr \\ *corresponding author: gauthier@ens2m.fr
}

\begin{abstract}
During microscale object manipulation, contact (pull-off) forces and non-contact (capillary, van der Waals, and electrostatic) forces determine the behaviour of the micro-objects rather than the inertial forces. The aim of this article is to give an experimental analysis of the physical phenomena at a microscopic scale in dry and liquid media. This article introduces a review of the major differences between dry and submerged micromanipulations. The theoretical influences of the medium on van der Waals forces, electrostatic forces, pull-off forces and hydrodynamic forces are presented. Experimental force measurements based on an AFM system are carried out. These experiments exhibit a correlation better than $40 \%$ between the theoretical forces and the measured forces (except for pull-off in water). Finally, some comparative experimental micromanipulation results are described and show the advantages of the liquid medium
\end{abstract}

\section{Keywords}

Micromanipulation, microrobotics, liquid medium, pull-off force, electrostatic force, hydrodynamic effect, van der Waals force, capillary force.

\section{Introduction}

The complexity of the microsystems is always higher and requires a lot of different materials and different microfabrication processes. Without micro-assembly technologies, it is more and more difficult to build microsystems and especially optical microsystems [1]. Consequently, the advent of new hybrid microsystems requires new micro-assembly technologies and methods. There are two main approaches in this domain: Self-assembly and robotic assembly. The first approach is useful for a very large production batch but the reliability stays low [2]. The second approach is more flexible and is relevant for a smaller production batch $[3,4]$.

Robotic micro-assembly tasks require firstly to be able to manipulate (to catch, to position, to release) microscopic objects whose typical size is included between one millimeter and one micrometer (micromanipulation).

The physical scale of micromanipulation is near to the lower limit of traditional mechanics. In general, the laws of Newtonian physics are still valid and the quantum effects neglected: The scale considered is 
thus at the boundary of two traditional spaces whose limits are not exactly known. The major difference with the macroscopic scale is indeed the results from the considered forces. The volume forces are negligible in respect to the surface forces for the microscopic objects [5-8]. These forces, whose effects are negligible on a macroscopic scale, modify drastically the contact mechanics and the interactions between the various media.

These surface forces may affect the micromanipulation task and especially the release of the microobject. The frontier generating the modification of the micro-object behaviour (from a behaviour dominated by surface forces rather than volume based forces) is a function of the material of the micro-gripper, object, and the surrounding medium. In most cases, this frontier corresponds to the specific dimension of the micro-object near 100 micrometers, and at the present time, no repeatable and reliable micromanipulator exists under this physical limit.

Most modelling of the micro-world is done in the dry medium (air or vacuum) [7, 9]. The liquid medium is not studied even through it could have a lot of advantages in micromanipulation of artificial objects under the limit of 100 micrometers. The objective of this work is to present the potential advantages of the liquid in artificial micro-object micromanipulation by means of theoretical and experimental forces analysis and first comparative pushing micromanipulations.

This article focuses on the theoretical and experimental comparison between both types of medium. However we focus this article on the experimental and theoretical analysis on micromanipulations in water, our general approach concerns liquids and not only water. The aim of this analysis is to show the potential relevance of submerged micro-assembly tasks. Further works will focus on the comparison between liquids to define the best liquid to perform micro-assembly.

The following section focuses on the theoretical impact of the medium on distance forces (van der Waals, electrostatic, capillary forces), contact forces (pull-off forces) and hydrodynamic forces. Thereafter, the measurements of distance and contact forces are presented and compared to theoretical values. The last section deals with comparative micromanipulation experimentations in dry and liquid media.

\section{Theoretical Analysis}

A lot of studies have been carried out on forces at microscopic scale. They use either classical models of forces at microscopic or nanoscopic scale (van der Waals, capillary, electrostatic forces) or theories of macroscopic contact (Hertz, JKR or DMT models). We propose a general approach by sorting out these forces considering the distinction whether there is contact or not. When there is no physical contact between two solids, the forces in action are called distance forces. According to the scientific literature in this domain $[8,10,11]$, the latter are electrostatic, van der Waals and capillary forces. In case of water medium, hydrophobic forces, steric forces and double-layer forces have to be considered too. When two solids are in contact, some object deformation appear which induce adhesion forces in the contact surface. In this case, we consider contact forces (usually denoted pull-off forces). Electrostatic or capillary effects can be added, but van der Waals forces are not considered anymore, because they are already involved in the pull-off term. In liquid the hydrodynamic effects have to be considered. Thus, the third type of forces presented is the hydrodynamic forces. 


\subsection{Surface Forces}

\subsubsection{Van der Waals Forces}

The van der Waals forces are a well-known interatomic interaction forces. For an interaction between a flat substrate (1) and a spherical object (2), the integrated van der Waals force is equal to:

$$
F_{v d w}(D)=-\frac{A_{12} R}{6 D^{2}}
$$

where $A_{12}$ is the Hamaker constant of the interaction (1-2), $D$ is the contact distance between (1) and (2) and $R$ is the radius of the spherical object (2).

Parameter $A_{12}$ usually takes values included in the interval $[0.4-4] \times 10^{-19} \mathrm{~J}[12-15]$. It is possible to obtain approximated values of $A_{12}$ by using the "combination laws", derived from the expression of $A_{12}$ introduced by Mac Lachlan in 1963 [16]: For two materials interacting in vacuum, $A_{12}$ is computed according to the constants $A_{i i}$ of each material:

$$
A_{12} \simeq \sqrt{A_{11} A_{22}}
$$

The Hamaker constant could be determined through the Lifshitz-van der Waals constant too [17]:

$$
A_{12}=\frac{3 H_{L V}}{4 \pi}
$$

where $H_{L V}$ is the Lifshitz-van der Waals constant.

For interaction of two materials in the presence of a third medium (3), the total force $F_{t}$ to considered is expressed by the extended DLVO theory (XDLVO) proposed by Xu and Yoon [18, 19]:

$$
F_{t}=F_{v d w}+F_{d l}+F_{h}
$$

The total force is the sum of the van der Waals force, the double-layer force and a third term which represents all other forces except van der Waals force and double-layer force, such as solvation, structural, hydration, hydrophobic, steric, fluctuation forces, etc.

The van der Waals force in a third medium is a function (1) of the Hamaker constant denoted $A_{132}$ estimated by:

$$
A_{132}=A_{12}+A_{33}-A_{13}-A_{23}
$$

Consequently, from (2), $A_{132}$ verifies:

$$
A_{132}=\simeq\left(\sqrt{A_{11}}-\sqrt{A_{33}}\right)\left(\sqrt{A_{22}}-\sqrt{A_{33}}\right)
$$

The repulsive double layer force $F_{d l}$ can be currently written as $[14,20,21]$ :

$$
F_{d l} \simeq 4 \pi R \epsilon_{3} \kappa_{3} \Phi_{1} \Phi_{2} e^{-\kappa_{3} D}
$$

where $\epsilon_{3}$ is the dielectric constant of the medium, $\Phi_{1}$ and $\Phi_{2}$ are the surface potentials of the sphere and the surface and $\kappa_{3}$ the Debye length of the medium. The repulsive double layer force $F_{d l}$ is typically greater than the van der Waals force between $D=1 \mathrm{~nm}$ to $D=10-20 \mathrm{~nm}$ [14]. This repulsive force is able to reduce the impact of the van der Waals force in this range. 
The third term represents notably the solvation forces which have typically significant impact at very small range lower than $10 \mathrm{~nm}$. In water, these forces are repulsive for hydrophilic surface and attractive for hydrophobic surface [14]. In case of hydrophilic surface these forces are able to reduce the impact of the van der Waals force.

Table 1 gives the values of Hamaker constant for some materials in vacuum and in water. The immersion is then able to reduce the value of the van der Waals force. However, this force has a short range (typically $<100 \mathrm{~nm}$ ) compared to the size of the object (greater than $1 \mu \mathrm{m}$ ). The impact of this force on the micro-objects behaviour is thus limited compared to the very long range of electrostatic interaction and contact forces.

\begin{tabular}{|l|c|c|}
\hline Materials & Vacuum & Water \\
\hline Gold & 40 & 30 \\
Silver & 50 & 40 \\
$\mathrm{Al}_{2} \mathrm{O}_{3}$ & 16.8 & 4.4 \\
Copper & 40 & 30 \\
\hline
\end{tabular}

Table 1: Values of Hamaker constant for some materials $A \times 10^{-20} J[22]$

\subsubsection{Electrostatic Forces}

The force applied by an electrostatic surface ( $\sigma$ surface charge density) on an electric charged particle $(q)$ is given by:

$$
F_{e}=\frac{q \sigma}{2 \varepsilon_{0} \varepsilon}
$$

where $\varepsilon$ and $\varepsilon_{0}$ are respectively the relative dielectric constant of the medium and the dielectric constant of the vacuum.

Comparison of dielectric constants between the water and the air is presented in Table 2. The water dielectric constant is more important than the air dielectric constant. So, in the same electrical charges configuration $(q, \sigma)$ electrostatic force is significantly reduced in water.

Moreover electrostatic perturbations observed in micromanipulation are caused by tribo-electrification. During a micro-assembly task, friction between manipulated objects induces electric charges on surface of the objects. The charge density depends on the tribo-electrification and conductivity of the medium. Effectively, a higher electric conductivity medium is able to discharge objects surfaces. The water, especially ionic water, has better electric conductivity than the air (Table 2). Consequently, charge density in water is reduced. The electrostatic force directly proportional to the charge density $\sigma$ is therefore reduced.

\begin{tabular}{|l|c|c|}
\hline Electric parameters & Air & Water \\
\hline Dielectric constant $\varepsilon$ & $\sim 1$ & 80.4 \\
Conductivity & $10^{-7}$ S. $m^{-1}$ & $>10^{-4}$ S. $m^{-1}$ \\
\hline
\end{tabular}

Table 2: Relative dielectric constant and electrical conductivity of air and water 
Both impacts of the immersion on electric properties of the medium (dielectric constant and conductivity) induce a reduction of electrostatic forces. In conclusion, electrostatic perturbations are highly reduced in water compared to the air.

\subsubsection{Capillary Forces}

Basically, the capillary forces arise in two ways: Either a liquid drop is put between two solids (e.g. a gripper and a component) that turns itself towards a meniscus (a liquid bridge), or a capillary bridge appears by condensation of the ambient humidity in the small cracks and pores made by two rough profiles brought together in contact.

In both cases, the situation can be described by a liquid bridge presented in Figure 1 characterised by a volume $V$, a liquid surface tension $\gamma$ and wettability properties defined by the contact angles $\theta_{1}$ and $\theta_{2}$. Most often the capillary forces are approximated by several formulations. With the assumptions that the contact angles are equal $\theta_{1}=\theta_{2}=\theta$, a constant volume and immersion height $(D)$ is small, capillary force between a plan and sphere (radius $R$ ) is equal to [14]:

$$
F_{c}=\frac{4 \pi R \gamma \cos \theta}{1+(D / d)}
$$

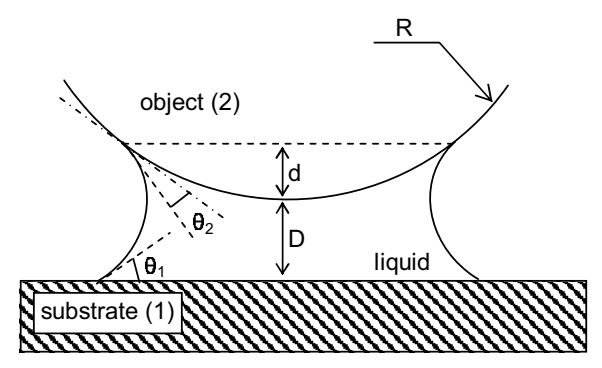

Figure 1: Liquid meniscus formation between a spherical object and a substrate.

This capillary force is induced by the surface between the liquid and the air near to the object. In liquid this surface disappears, so this force is cancelled in liquid medium.

\subsection{Contact Forces}

The pull-off force represents the force necessary to break the contact surface between two objects. In case of a sphere (radius $R$ ) on a planar surface, pull-off force $P$ is approximately given by $\mathrm{JKR}^{1}$ (for the lower boundary) or $\mathrm{DMT}^{2}$ (for the higher boundary) contact models [23, 24]:

$$
\frac{3}{2} \pi R W_{12} \leq P \leq 2 \pi R W_{12}
$$

where $W_{12}$ is the work of adhesion between both objects (1) and (2).

In the air, the work of adhesion is expressed by [25]:

$$
W_{12}=\gamma_{1}+\gamma_{2}-\gamma_{12} \simeq 2 \sqrt{\gamma_{1} \gamma_{2}}
$$

\footnotetext{
${ }^{1}$ Johnson, Kendall and Roberts [23]

${ }^{2}$ Derjaguin, Muller and Toporov [24]
} 
where $\gamma_{12}$ is the interfacial energy and $\gamma_{1}, \gamma_{2}$ are the surface energy of both objects.

According to [26], the Maugis elasticity parameter $\lambda$ can be used to choose the most appropriate contact model for a given case. This parameter is expressed for an interface between two bodies (1) and (2) with:

$$
\lambda=2 \sigma_{0}\left(\frac{R}{\pi W_{12} K^{2}}\right)^{\frac{1}{3}}
$$

where $K$ is the equivalent elastic modulus, calculated using the both Poisson's ratios $\mu_{1}, \mu_{2}$ and both Young's modulus $E_{1}, E_{2}$ :

$$
K=\frac{4}{3}\left(\frac{1-\mu_{1}^{2}}{E_{1}}+\frac{1-\mu_{2}^{2}}{E_{2}}\right)
$$

The parameter $\sigma_{O}$ is defined by:

$$
\sigma_{0}=\frac{W_{12}}{h}
$$

where $h \simeq 10^{-10} \mathrm{~m}$.

Using $\lambda$, the pull-off force can be estimated with:

$$
\begin{array}{rll}
\lambda<0.1 & \Longrightarrow \text { DMT model: } & P=2 \pi R W_{12} \\
\lambda>5 & \Longrightarrow \text { JKR model: } & P=\frac{3}{2} \pi R W_{12} \\
0.1<\lambda<5 & \Longrightarrow \text { Dugdale model: } & P=\left(\frac{7}{4}-\frac{1}{4} \frac{4.04 \lambda^{\frac{1}{4}}-1}{4.04 \lambda^{\frac{1}{4}}+1}\right) \pi R W_{12}
\end{array}
$$

Moreover, in case the objects are submerged in medium (3), the surface energy, denoted $W_{132}$, required to separate two objects (1) and (2) submerged in a medium 3 is given by:

$$
W_{132}=W_{12}+W_{33}-W_{13}-W_{23} \simeq \gamma_{13}+\gamma_{23}-\gamma_{12}
$$

For example, in case of a $\mathrm{SiO}_{2}-\mathrm{SiO}_{2}$ contact $\left(\gamma_{\mathrm{SiO}_{2}}=290 \mathrm{~mJ} \cdot \mathrm{m}^{-1}\right.$ [22]), the theoretical surface energies in air and in water are (from (11), (16)):

$$
W_{12}=580 m J . m^{-1} \quad W_{132}=146 m J . m^{-1}
$$

In this example, the pull-off force is reduced in water compared to the air. Usually, solid state surface energies are around $1000 \mathrm{~mJ} . \mathrm{m}^{-1}$ and the theoretical pull-off reduction is around $50 \%$ to $80 \%$.

\subsection{Impact of the Hydrodynamic Forces on the Micro-objects Behavior}

In this section the impact of the hydrodynamic forces on the behaviour of micro-objects is described. In the micro-world, the Reynolds number which characterises the liquid flow is usually very low $(<1)$. The flow is thus highly laminar. In case of a micro-object placed in an uniform liquid flow, the Stokes law directly gives the hydrodynamic force applied on the object. This law is valid when the flow Reynolds number is lower than 1 and can be extrapolated to Reynolds number lower than 10 with a good approximation. 
The Stokes law defines the force applied on an object in a uniform flow of fluid defined by a dynamic viscosity $\mu$ and a velocity $V$ :

$$
\vec{F}_{\text {hydro }}=-k \cdot \mu \cdot \vec{V}
$$

where $k$ is a function of the geometry. In case of a sphere with a radius $R, k$ is defined by

$$
k=6 \pi R
$$

Table 3 gives the values of dynamic viscosity $\mu$ of both water and air. Then the hydrodynamic force proportional to the dynamic viscosity highly increases in a submerged medium.

\begin{tabular}{|l|c|c|}
\hline Dynamic viscosity & Water & Air \\
\hline$\mu\left[k g \cdot \mathrm{m}^{-1} . \mathrm{s}^{-1}\right]$ & $10^{-3}$ & $18.510^{-6}$ \\
\hline
\end{tabular}

Table 3: Dynamic viscosity of water and air, $T^{\circ}=20^{\circ} \mathrm{C}$

As inertial effects are very small in the micro-world, micro-objects accelerations are usually very high. In this way, micro-object velocity is able to increase in a very short time. Consequently, micro-objects can reach high velocity, and object trajectory could be difficult to control especially in case of a visual feedback. In fact, the object can jump rapidly out of the field of view and this induces its loss. So, in most cases, velocity limitation in the submerged micro-world does not depend on inertial physical limitation but on hydrodynamic physical limitation. From this, a liquid medium is able to reduce maximal micro-objects velocity [27]. Consequently, the increasing of hydrodynamic force is able to limit the maximal velocity of the objects and thus significantly reduces the loss of micro-objects.

However, movements of liquid induced by the movement of the effector are able to lead to significant hydrodynamic force on micro-objects. Consequently the hydrodynamic force induces a limitation of the maximum velocity of the effector to avoid disturbance on the micro-object position. Nevertheless, experimentally the maximum velocity of the effector can stay high (eg. $1 \mathrm{~mm}^{-\mathrm{s}^{-1}}$ ) compared to the typical size of the object manipulated $(50 \mu \mathrm{m})$.

In conclusion, contact, non contact and hydrodynamic force were presented in both liquid and dry media. This analysis shows the reduction of contact and non contact forces in liquid compared to the air. As these effects are able to perturb the micromanipulation tasks, the use of a liquid could improve the efficiency of micromanipulation. Moreover, the increase of the hydrodynamic effects are beneficial on the micro-objects behaviour during their micromanipulation. Thus, the theoretical study shows the interest of submerged media for such tasks.

\section{Forces Measurement}

To analyse the validity of the micro-force modelling, some experimental force measurements are necessary. This part deals with the presentation of the micro-force measurement device and the comparison between theoretical and experimental values.

\subsection{AMIS System}

The micro-forces are measured by a specifically developed system called AMIS (AFM based MIcromanipulation System). This system is based on a standard Atomic Force Microscope (AFM) and a 3D 
micromanipulation system which allows large displacement (which is not usually the case in a standard AFM). Consequently, this system is able to measure contact force and interaction distance like a standard AFM but also very long interaction distance (up to 1 micrometer). This second functionality will notably be used to measured electrostatic forces.

\subsubsection{Description}

A view of AMIS is given in Figure 2. This system is based on an AFM where the cantilever can be moved in three perpendicular directions $\mathrm{XYZ}^{3}$ by a piezotube with $\mathrm{X}, \mathrm{Y}$ and $\mathrm{Z}$ strokes of respectively 45 $\mu \mathrm{m}, 45 \mu \mathrm{m}$ and $4 \mu \mathrm{m}$. The measured hysteresis of the piezotube in the $\mathrm{Z}$ directions is $16 \%$. Three linear micropositioning stages are also used for the studied sample motions on longer strokes in XYZ directions: $15 \times 15 \times 15 \mathrm{~mm}^{3}$, with a repeatability of $0.5 \mu \mathrm{m}$. All these motions can be controlled in automatic mode or in manual mode, notably by using a force-feedback joystick. This joystick applies in real time to the operator the bending and torsion effects measured on the cantilever. These strains are measured by a photodiode (which delivers a corresponding voltage $V_{m}$ ).

Finally two microscopes with CCD cameras give visual information in real time on respectively vertical and lateral views (see Figure 2): The first is fixed under the glass sample support and the second is placed laterally. Then the first one gives the position of the cantilever tip in the sample plan. The second one is used to estimate the vertical distance between the tip and the sample plan.

The AMIS system is used for surface forces measurements as well as for micromanipulation. In this last case the used end-effector of AMIS is the cantilever of the AFM.

In this paper, the cantilever is a silicon one with $350 \mu \mathrm{m}$ in length, $35 \mu \mathrm{m}$ in width and $2 \mu \mathrm{m}$ in thickness. Its tip has a curvature radius of less than $10 \mathrm{~nm}$ and a height of $10 \mu \mathrm{m}$ (see Figure $2 \mathrm{~b}$ ). The resonance frequency $f$ and the stiffness $k$ of the tip are respectively:

$$
\begin{aligned}
& f=7.4 k H z \\
& k=0.03 N . m^{-1}
\end{aligned}
$$

The usual precision of stiffness measurements $k$ through the resonance frequency $f$ is around $10 \%$.

The noise measured on the photodiode induces an uncertainty on the force measurement experimentally estimated lower than $400 p N$. The piezotube displacement step used in the force-distance curves presented is $15 \mathrm{~nm}$. During the contact phase, this step corresponds to a contact force increment lower than $500 \mathrm{pN}$. Thus, the repeatability of the pull-force measurement is estimated lower than $1 \mathrm{nN}$.

\subsubsection{Measurement Method}

Our set-up is used here for the production of experimental force-distance curves based on the realtime measurement of the AFM cantilever bending. A force-distance curve is a quasi-static trajectory which corresponds to an "approach and retract" cycle between the cantilever and the sample (in the Z direction). Depending on the required stroke these motions are actuated by the piezotube as well as the sample vertical axis. An example of a typical force-distance curve (eg. copper substrate) is presented in Figure 3. According to this Figure, the successive steps of the measurement are:

- Step 1: The approach phase starts. The cantilever is in its free state, i.e. without any bending.

- Step 2: At a sufficiently small distance of its tip to the sample, the cantilever begins to bend in a concave shape: the distance forces start to act (electrostatic and van der Waals forces).

\footnotetext{
${ }^{3}$ the $\mathrm{Z}$ axis designing the vertical direction.
} 


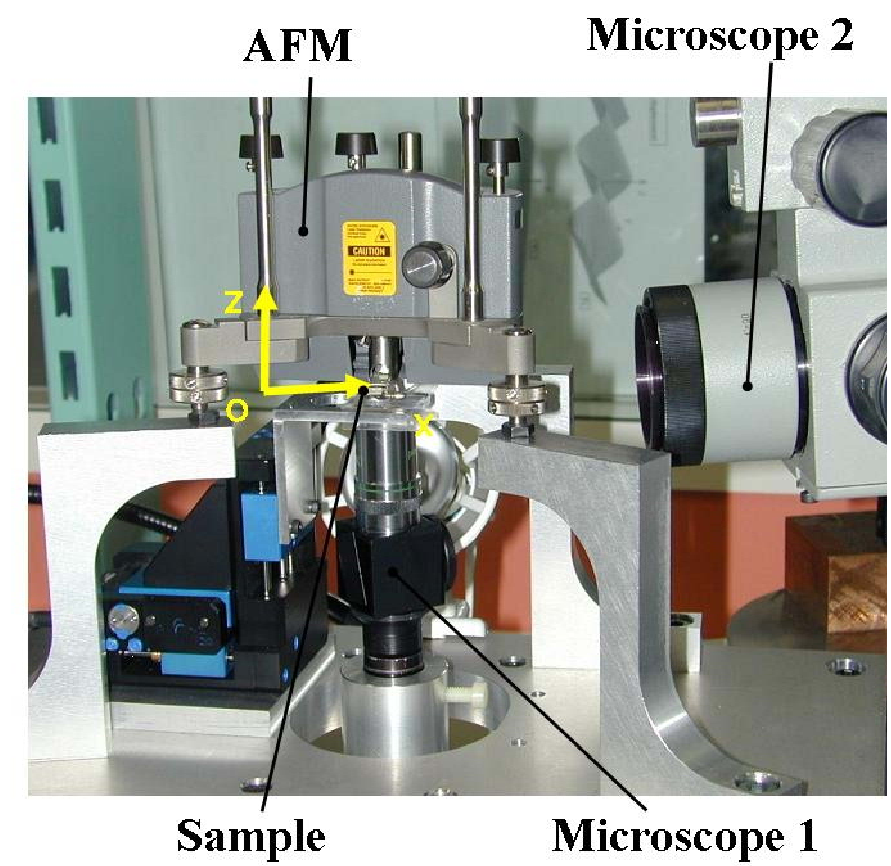

(a) View of the experimental force measurement system.

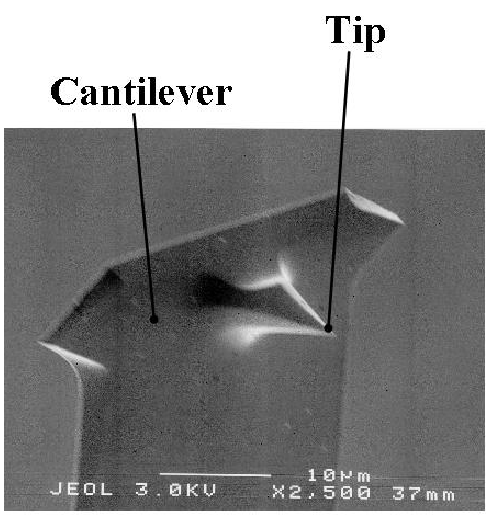

(b) View of the tip.

Figure 2: AFM based MIcromanipulation System (AMIS).

- Step 3: The contact tip-sample is now established.

- Step 4: Continuing the approach movement, the cantilever comes back to its free state (no bending) then, because of the contact, bends according to a convex shape. This phase is then stopped when a maximal force is reached.

- Step 5: The taking away phase starts (an hysteresis can usually be observed).

- Step 6: Continuing this phase, the cantilever goes first to its free state then bends again according to its concave shape. The contact tip-sample is still maintained in this phase. Depending on the magnitude of the pull-off force this bending could be very high.

- Step 7: The cycle ends when the elastic return force becomes higher than the pull-off force. Then the contact is broken and the cantilever returns to its free state.

For each force-distance curve, the force measurement is performed several times (a minimum of 3 times). We evaluated the variations (not given in this article) which showed a good repeatability compared to the force measured and compared to the difference between experimental and theoretical values.

First the chosen sample is placed in the AMIS system, the operator starts the application and the "approach and retract" cycle is then executed automatically. The acquired data is the cantilever bending $\delta$, i.e. the voltage measured from the photodiode $V_{m}$, according to the relative vertical motion between the cantilever and the sample. Then an adequate processing is done to obtain the measured force $F$ : First the cantilever bending $\delta$ is a function of the measured voltage $V_{m}$ (equation (19)).

$$
\delta=C \cdot V_{m}
$$


A specific identification procedure is used to estimate the coefficient $C$. Secondly, the measured force is a function of the cantilever stiffness $k$ and the bending $\delta$ :

$$
F=k \cdot \delta=k \cdot C \cdot V_{m}
$$

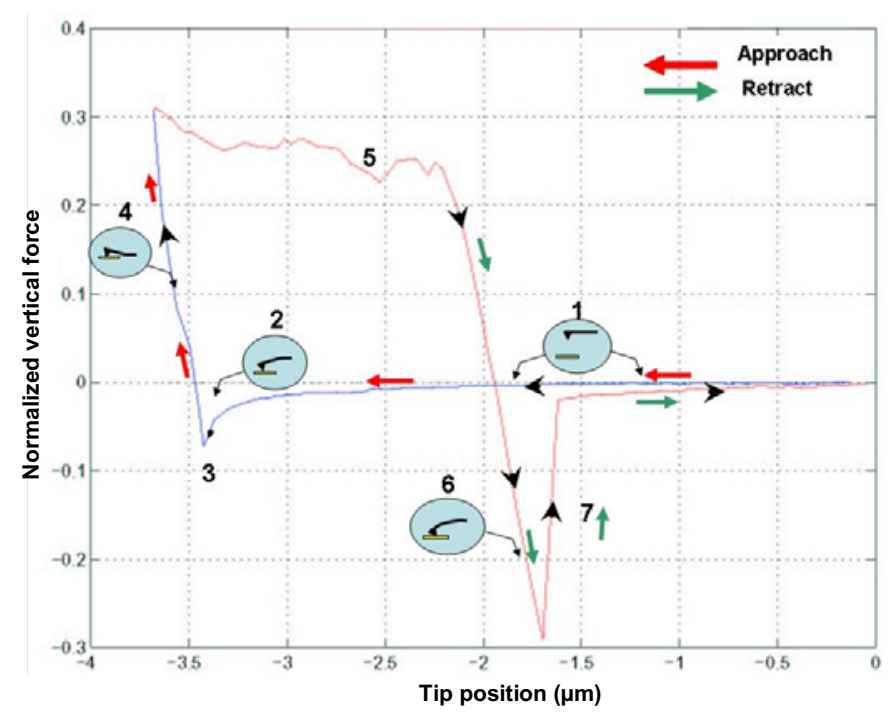

Figure 3: Example of force-distance curve (eg. copper substrate).

\subsection{Experimental Measurements}

\subsubsection{Pull-off Forces}

First, AMIS is used to study the pull-off force. These experiments are carried out with polystyrene (PS) and glass substrates.

The pull-off force is measurable on the experimental force-distance curves when the breaking load between the AFM tip and the substrate appears (mark (1) in the Figure 4). From these curves (Figure 4 ), an experimental value of the pull-off forces for both interactions is measured. These values are estimated as:

$$
\begin{aligned}
P_{\text {silicon-PS }}^{\text {measured }} & =26 n N \\
P_{\text {silicon-glass }}^{\text {measured }} & =35 n N
\end{aligned}
$$

From equation (11), (12), (15) and physical properties described Table 4, theoretical pull-off forces can be calculated:

$$
\begin{aligned}
P_{\text {silicon-PS }}=28 n N & (\lambda=0.33) \\
P_{\text {silicon-glass }}=49 n N & (\lambda=0.54)
\end{aligned}
$$




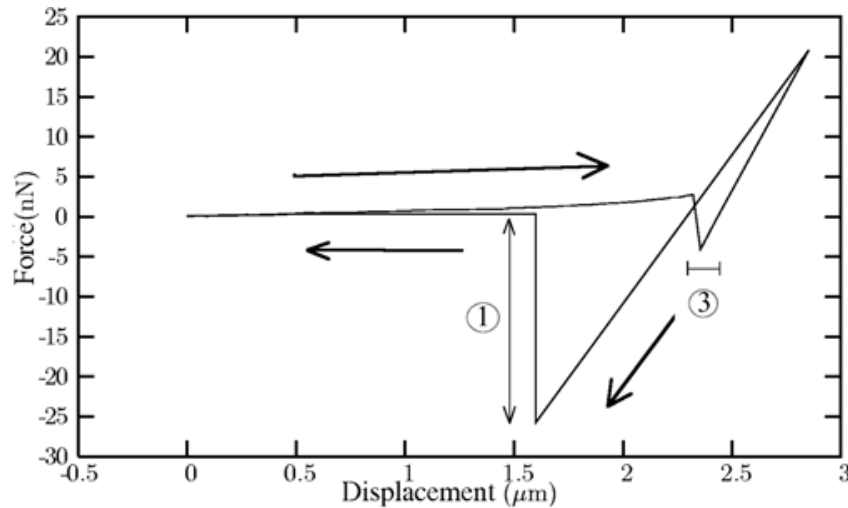

(a) Interactions between AFM tip and a PS substrate.

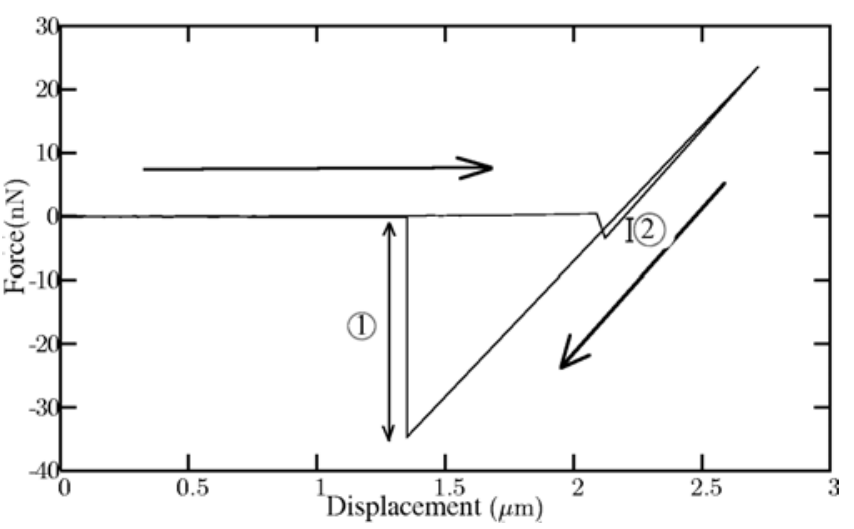

(b) Interactions between AFM tip and a glass substrate.

Figure 4: Force-distance curves in air.

These values (24)-(25) fit very closely to the measurements (22)-(23). Hence, theoretical estimation of pull-off forces can generally be trusted when no direct measurements are possible.

In order to analyse the influence of the environment, pull-off force measurement was done in aqueous medium. Figure 5 describes the force-distance curve of a silicon-glass interface in water. The experimental pull-off force is thus estimated as:

$$
P_{\text {silicon-water-glass }}^{\text {measured }}=5.5 \mathrm{nN}
$$

From equation (16), (12), (15) and physical properties described Table 4, theoretically calculated pull-off force is then:

$$
P_{\text {silicon-water-glass }}=16.0 \mathrm{nN}
$$

Pull-off force induces adhesion effects in a micromanipulation task. Consequently the significant reduction of the pull-off force in liquid is able to reduce adhesion perturbations in submerged micromanipulations.

\begin{tabular}{|l|c|c|c|c|}
\hline Material & $\gamma$ & $A$ & $\nu$ & $E$ \\
\hline Unity & $\mathrm{mJ} . \mathrm{m}^{-2}$ & $\times 10^{-20} \mathrm{~J}$ & - & $\mathrm{GPa}$ \\
\hline Silicon & 1400 & 26 & 0.17 & 140 \\
Polystyrene (PS) & 36 & 7.9 & 0.35 & 3.2 \\
Glass & 170 & 6.5 & 0.25 & 69 \\
\hline
\end{tabular}

Table 4: Physical properties of the materials used in the experiments.

\subsubsection{Van der Waals Forces}

Van der Waals forces induce attraction in the approach phase of the AFM tip. On the force-distance curves presented in Figure 4, the attraction phenomena is observable in the approach phase (mark (2) and (3) in Figure 4). Though some works suggested different origins to the behaviour, a traditional 


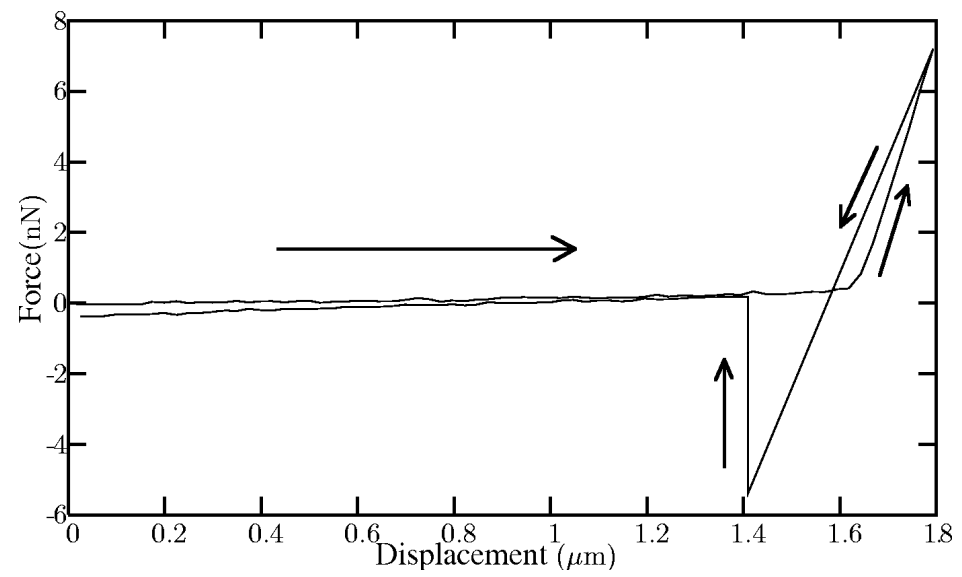

Figure 5: Force-distance curve for an interaction between the cantilever and a glass substrate in an aqueous medium.

approximation is to consider only van der Waals interaction $[28,29]$. These forces are thus estimated at:

$$
\begin{aligned}
F_{\text {vdw silicon-glass }}^{\text {measured }} & =-5.1 n N \\
F_{\text {vdw silicon-PS }}^{\text {measured }} & =-4.0 n N
\end{aligned}
$$

With the assumption of the contact distance $D_{0}=0.17 \mathrm{~nm}$ [25], the values of Hamaker constants of the interfaces are deduced from measurements:

$$
\begin{aligned}
A_{\text {silicon-glass }}^{\text {measured }} & =8.4 \times 10^{-20} \mathrm{~J} \\
A_{\text {silicon-PS }}^{\text {measured }} & =6.6 \times 10^{-20} \mathrm{~J}
\end{aligned}
$$

These measured values can be compared with the theoretical values obtained with (2):

$$
\begin{aligned}
A_{\text {silicon-PS }} & =14 \times 10^{-20} \mathrm{~J} \\
A_{\text {silicon-glass }} & =13 \times 10^{-20} \mathrm{~J}
\end{aligned}
$$

The errors are more significant because it is difficult to determine the various phenomena [30]. Nevertheless this estimation seems to give a realistic value, in order to estimate these forces via the Hamaker constants. In case of a liquid medium (Figure 5), the influence of the van der Waals force in the approach phase was not perceptible by our system. Its influence thus seems negligible in aqueous environment.

A significant remark is that the range of van der Waals forces is of the order of one hundred nanometers for all experiments carried out. The range of this force is extremely small compared to the dimensions of the micro-objects. Thus, the impact of this non contact force on micro-objects behaviour seems to be negligible.

\subsubsection{Electrostatic Forces}

This part deals with the electrostatic forces in case of contact with conductors and insulators. AFM tip is made of silicon and is grounded. The first experiment describes a contact with a gold substrate 
(Figure 6). Comparative electrostatic force measurements were done on grounded and non grounded gold surface. These experimentations clearly show that the electrostatic force (marks 2 in Figure 6 ) is reduced when the substrate is grounded. On a non grounded substrate, the electrostatic forces appears at a very significant separation distance (mark 1 in Figure 6a) compared to the other forces (ten micrometers).

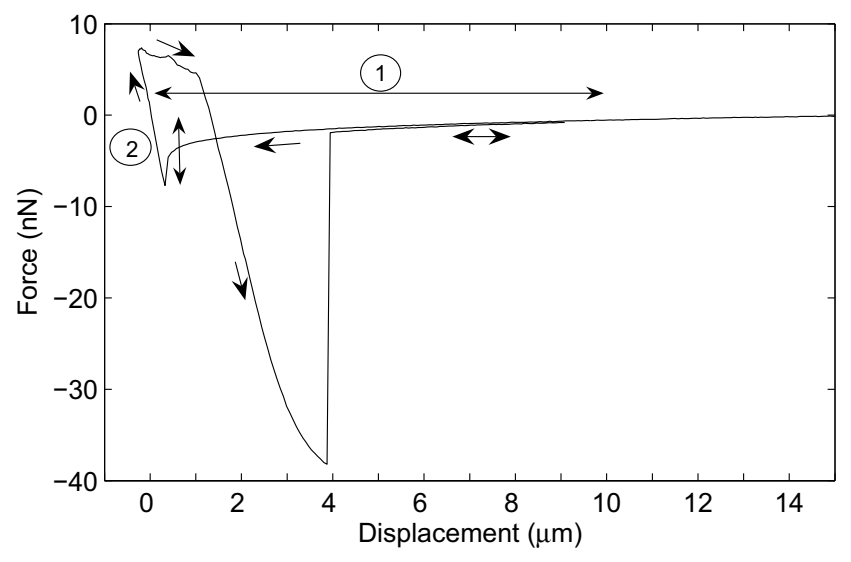

(a) Non grounded substrate.

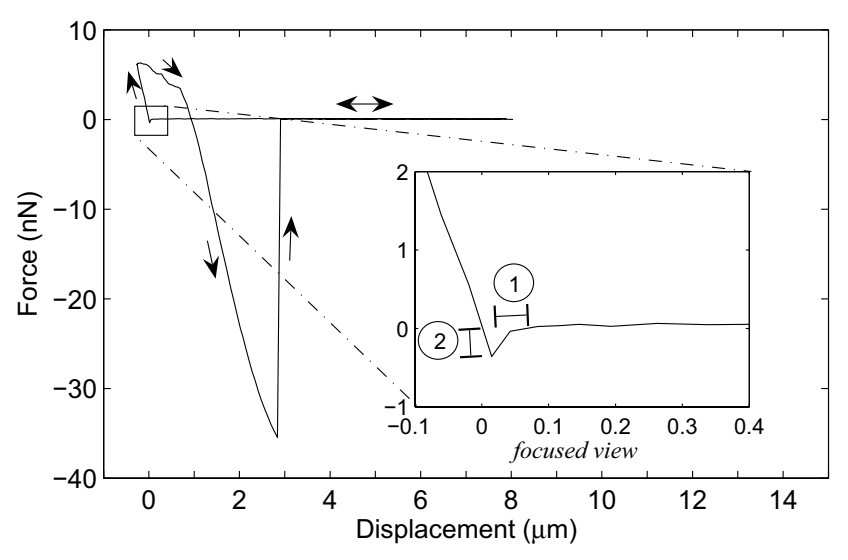

(b) Grounded substrate.

Figure 6: Force-distance curves in air with a gold substrate.

The second study is led on an insulator, PS substrate. The results are done in Figure 7 . In the same way, to avoid this force, the substrate is cleaned with distilled water. The curve obtained is then represented on the Figure $7 \mathrm{~b}$. The electrostatic force is clearly reduced after charge cleaning (marks 2 in figure 7). In the first case, the interaction distance of the electrostatic (marks 1 in figure 7a) which is about ten micrometers is larger than the interaction distance of the other forces again. The modification of the pull-off force between both cases presented figure 7 has not been studied. It could be explained by capillary forces induced by residual water after cleaning.

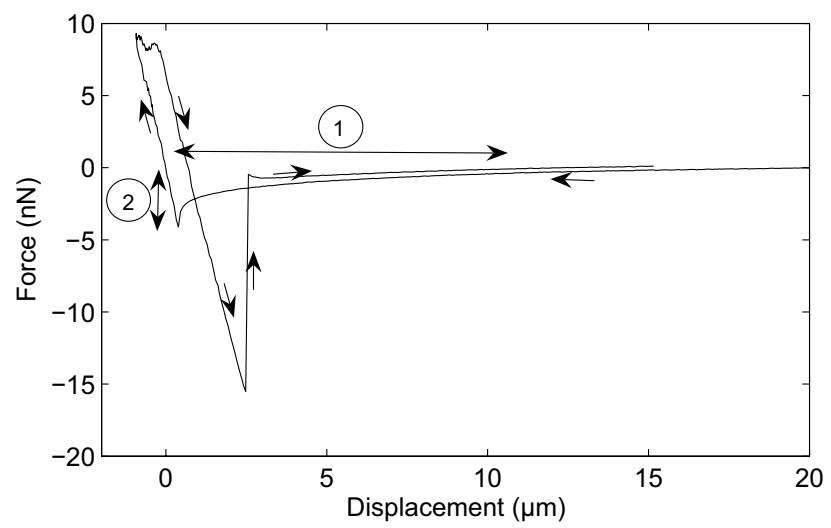

(a) Substrate without charges cleaning.

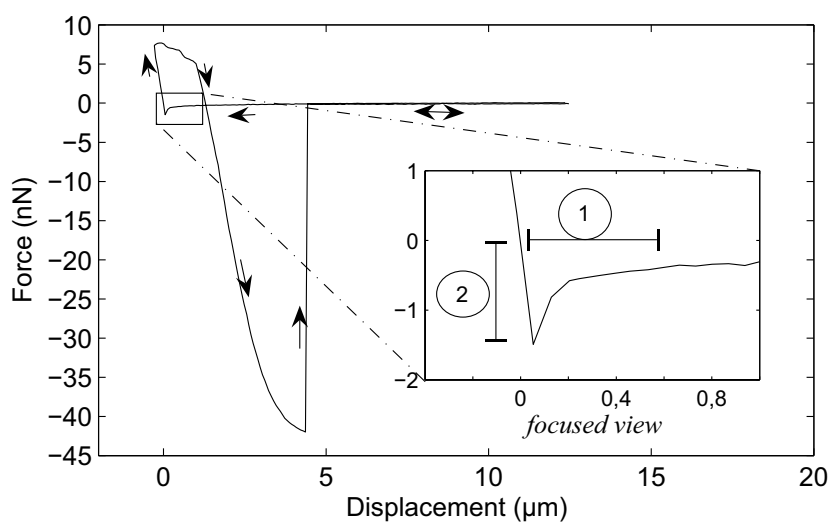

(b) Substrate after cleaning the substrate with distilled water.

Figure 7: Force-distance curves with a polystyrene substrate. 
To illustrate electrostatic perturbations, a third experimental study was done: The approach of the AFM cantilever with a copper substrate initially charged with a $2 \mathrm{~V}$ voltage. The approach curve of the AFM cantilever is then drastically modified (see Figure 8). The cantilever is periodically attracted by the substrate and release due to electrostatic effects. The attraction is induced by the long range of the electrostatic forces while the release is obtained by a local discharge of the substrate induced by the contact with the micro-tip. Moreover, tip effects can be observed, making difficult any identification. In the same way, this phenomenon disappears as soon as the substrate is grounded.

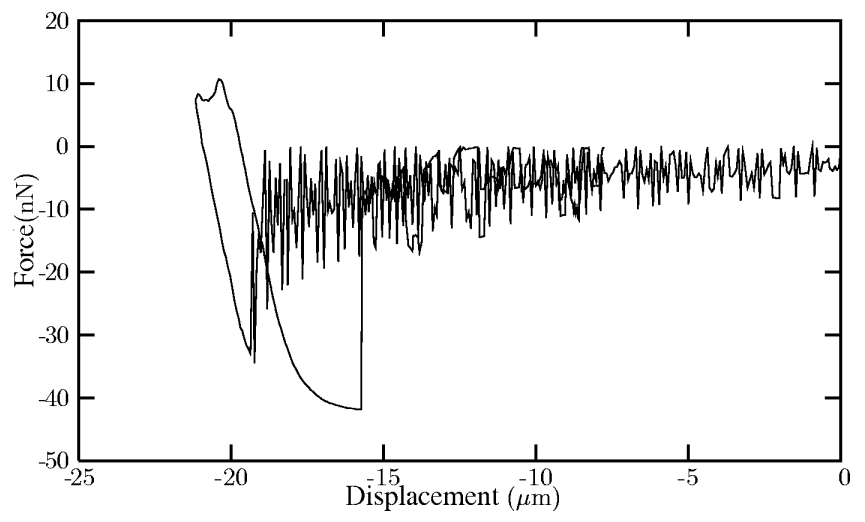

Figure 8: Electrostatic perturbations measured by AMIS.

Electrostatic forces are efficient in long range, starting at $10 \mu \mathrm{m}$ and have the highest modules of the distance forces. As the charge density of a micro-object is not exactly known, the values of the electrostatic forces in a real system are hard to model. The reduction of the electrostatic perturbations is thus a key point to perform repeatable and precise micromanipulations. In dry medium, the cancellation of electrostatic effects can be obtained by grounding for conductor or by using distilled water for insulator. In liquid, i.e water, the electrostatic effects are highly reduced (section 2.1.2). In fact, no electrostatic forces were measured in water.

The force measurements performed with the AMIS device prove a relatively good correlation between the micro-force models and the experimental forces. Moreover, the advantages of the liquid presented in section 2 , is confirmed by the experimental forces measurement.

\subsubsection{Capillary Forces}

For micromanipulation applications, the capillary forces can represent a key parameter. Two interactions are studied with our system by placing a water droplet on PS and glass substrates. As the height of the droplet exceeds the maximum stroke of the AFM probe actuator, only the table motion is possible. In this case force-distance curves are reversed in terms of displacement, and this motion reduces displacement precision.

This force appears only when the AFM probe actually is not in contact with the water droplet. This force can thus be estimated using the following contact angles [31]:

$$
\theta_{\text {glass }}=37^{\circ} \quad \theta_{\mathrm{PS}}=67^{\circ}
$$




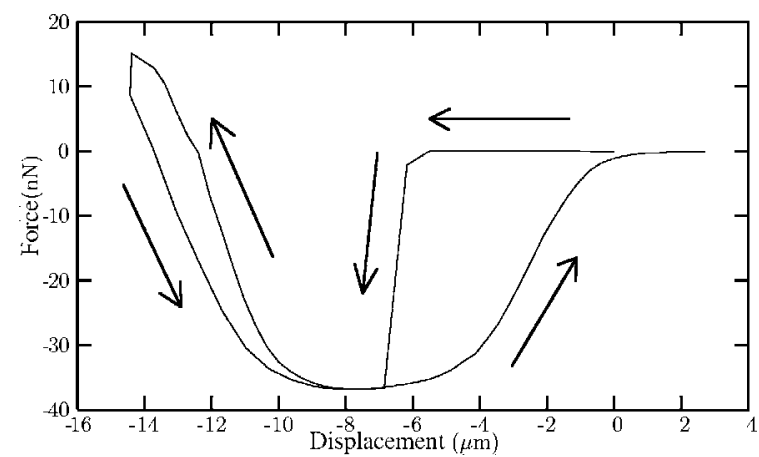

(a) On a PS substrate.

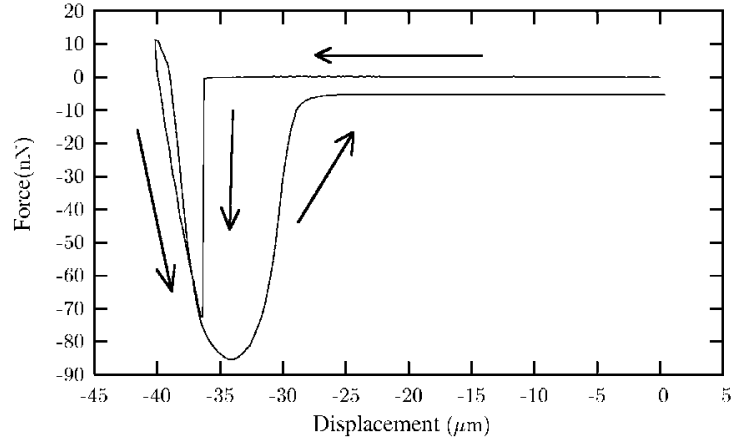

(b) On a glass substrate.

Figure 9: Force-distance curves for interactions between the AFM tip and a water droplet.

The measured forces are about:

$$
\begin{gathered}
F_{\mathrm{c} \text { silicon-glass }}^{\text {measured }}=72 n N \\
F_{\text {c silicon-PS }}^{\text {measured }}=43 n N
\end{gathered}
$$

The theoretical forces can thus be calculated from the equation (9):

$$
\begin{aligned}
F_{\text {c silicon-glass }} & =73 n N \\
F_{\text {c silicon-PS }} & =36 n N
\end{aligned}
$$

Experimental and theoretical values are near. The model is thus a good way to estimate this effect in micromanipulation.

In conclusion, the measurements of the non contact and contact forces generally show a good correlation between the theoretical models and the experiments. The correlation between the theoretical forces and the measured forces is better than $40 \%$ (except for pull-off in water). The measurement of the reduction of the pull force in water, and the cancellation of the electrostatic perturbations confirm the theoretical analysis. The interest of the submerged micromanipulation is thus confirmed by the force measurements.

\section{Micromanipulations in Dry and Liquid Media}

The purpose of this last part is to show the experimental interest of the submerged micromanipulation compared to the dry micromanipulation.

An automatic micro-assembly task requires firstly the development of reliable micromanipulation methods. At the present, the reliability in micromanipulation is one of the greatest keypoints. As the release of a micro-object is a critical and unreliable step, we focus our experimentations on the impact of the liquid on the release. In most cases, micromanipulation tasks are performed either by pushing or by gripping.

Our approach was tested on two micromanipulation stations: Manipulation with a piezoelectric microgripper, and manipulation by pushing with a glass microtip. Both devices are able to characterise liquid influence on each kind of classical micromanipulation. 


\subsection{Gripping Micromanipulation}

The first device used to compare dry and submerged micromanipulations is a micromanipulation station (Figure 10a) which includes a piezoelectric microgripper [32,33]. In this device, the substrate is fixed and the microgripper is moved with an X-Y-Z micropositioning motorised stages (3x M-111-1DG from PI company). The microgripper end-effector is made of electroplated nickel and its height measures 200 micrometers. Two views are used to usually observe the micromanipulations. The vertical view is obtained by a microscope with a CCD camera. The lateral view which displays the vertical position of the microgripper is done with a small CCD camera.

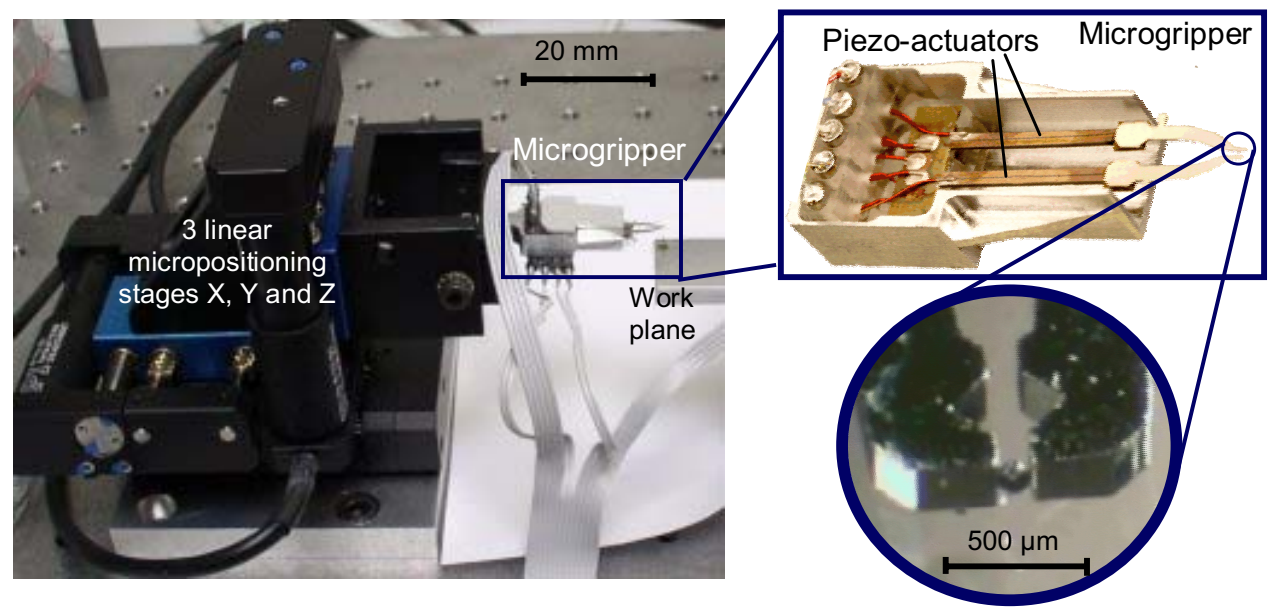

Figure 10: Gripping micromanipulation device.

To show the interest of liquid medium in microrobotics, pick-and-place micromanipulations in air and in water were tested. Figure 11 represents a lateral view of two attempts to release a micro-object $(100 \mu \mathrm{m})$ in both media. The manipulations are completely similar and are obtained with the same type of object, microgripper and substrate. The differences between both images are induced by the water refraction.

During these comparative experimentations, it clearly appears that the adhesion phenomena are less important in water than in air. The example given in Figure 11(a) shows that the object stays in contact with the microgripper in air, while the object is released in water (Figure 11(b)).

This example of comparative micromanipulations shows the influence of the medium on the adhesion forces and the interest of submerged micromanipulation tasks.

\subsection{Pushing Micromanipulation}

The second micromanipulation strategy compared in dry and liquid medium is the micro-objects pushing. A glass microtip is used to push micro-objects in water and in air. The substrate is placed on a X-Y- $\theta$ micropositioning device (M-111-1DG and M-038-DG from PI company) while the glass tip stays immobile under the microscope (Figure 12(b)). Microscope, micropositioning stage and microtip are placed on an anti-vibrations table. The stage motion is controlled by a computer. The micromanipulation tele-operation is performed with a CCD camera added on the microscope. Typical view range is $1000 \mu \mathrm{m} \times 700 \mu \mathrm{m}$. The end-effector is a cylindrical glass microtip with a diameter of $100 \mu \mathrm{m}$. The manipulated micro-objects are polystyrene (PS) microballs with a diameter of $50 \mu \mathrm{m}$. The substrate is 


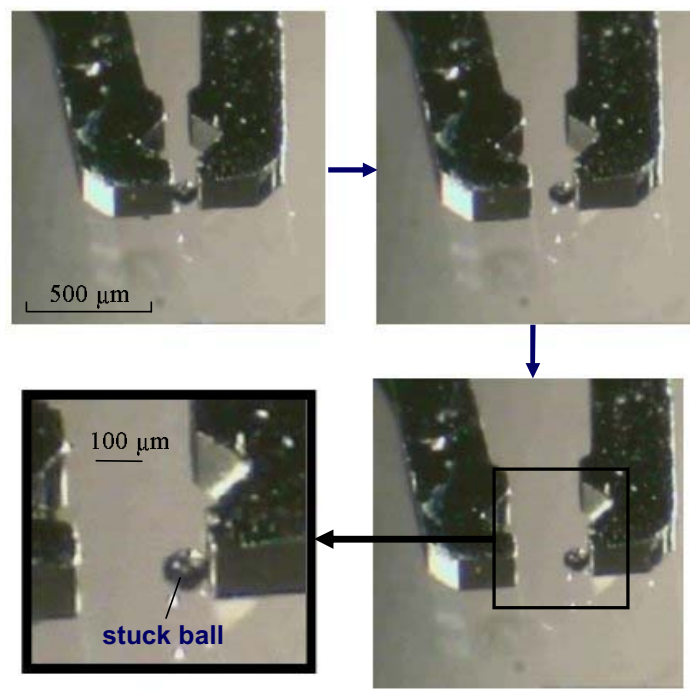

(a) Micro-object $(100 \mu m)$ release attempt in air.

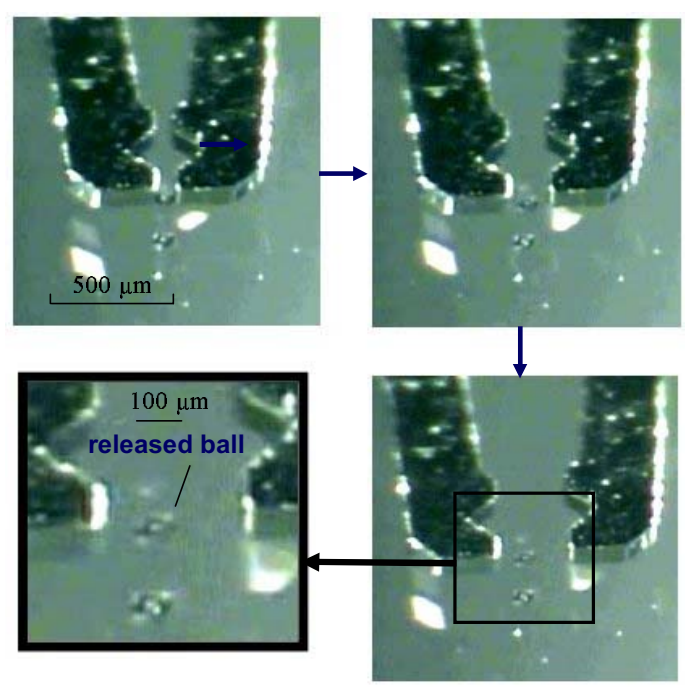

(b) Micro-object $(100 \mu \mathrm{m})$ release in water.

Figure 11: Experimental comparison between dry and liquid media: $100 \mu \mathrm{m}$ ball micromanipulations with the same microgripper.

also in polystyrene.

Comparison between PS ball micromanipulations in water and in air was performed with the pushing micromanipulation station. As the impact of the perturbations (electrostatic charges, adhesion forces) is significant, the micromanipulation results are not deterministic. We choose to analyse our experimental results by means of statistic analysis, and the pushing operations were classified in four different groups:

- Normal release: No adhesion effects are visible on the micro-object. The micro-object stays immobile during the release.

- Release and jump: During the pushing the micro-objects is propelled in a short time in a new position. This jump induces the release of the micro-object.

- Adhesion and release: When the glass microtip moves back, the micro-object stays attached to the glass tip. Thus the micro-object is not released. The glass tip is moved back and forth three times to try to release the micro-object, and the micro-object is released during these three attempts.

- Adhesion and failure: The micro-object is attached to the microtip (by adhesion). After three release attempts, the micro-object keeps attached. We consider this state as a failure and the action on an external tip is necessary to detach the micro-object.

The objective of the experimental micromanipulation was to write the word "LAB" as described in Figure 12(b). This task needs a large number of teleoperated micropushing operations. During micromanipulation, every operation was classified as presented in Table 5. The experimental micromanipulation was performed in air and in water to compare micropushing operation differences.

The first important difference lies in jumps which never occur in liquid. The jump phenomenon is effectively reduced by the hydrodynamic forces. From an automation point of view, the jump phenomenon 


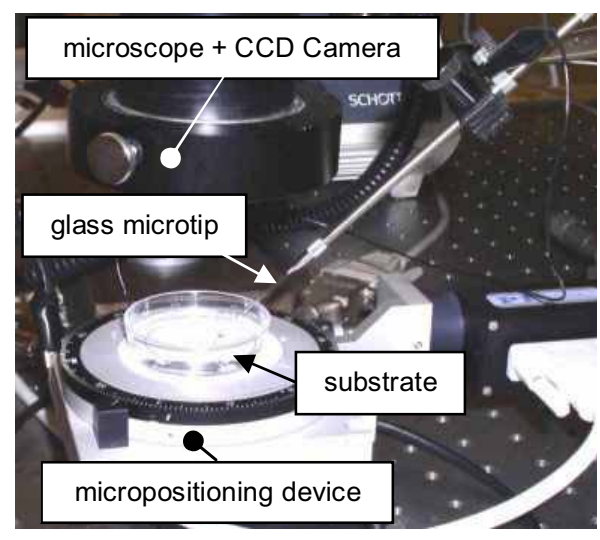

(a) Experimental device.

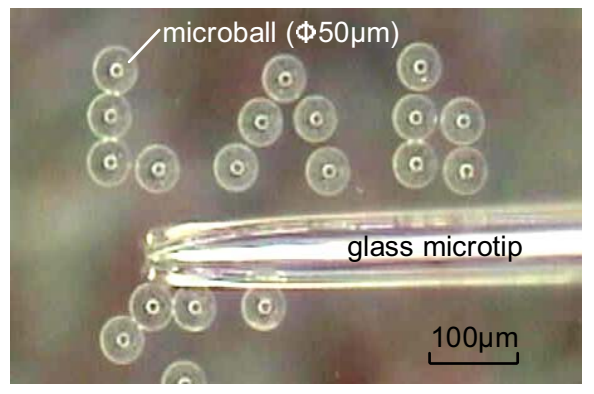

(b) Micromanipulation experimentation in water.

Figure 12: Pushing micromanipulation experimental device and micromanipulation experimentation.

\begin{tabular}{|l|c|c|c|c|c|}
\hline Medium & $\begin{array}{c}\text { Number } \\
\text { of } \\
\text { of tests }\end{array}$ & $\begin{array}{c}\text { normal } \\
\text { release }\end{array}$ & $\begin{array}{c}\text { release } \\
\text { and } \\
\text { jump }\end{array}$ & $\begin{array}{c}\text { adhesion } \\
\text { and } \\
\text { release }\end{array}$ & $\begin{array}{c}\text { adhesion } \\
\text { and } \\
\text { failure }\end{array}$ \\
\hline Air & 119 & $51 \%$ & $10 \%$ & $36 \%$ & $3 \%$ \\
Water & 97 & $67 \%$ & $0 \%$ & $33 \%$ & $0 \%$ \\
\hline
\end{tabular}

Table 5: Comparison of teleoperated micropushing operations in air and in water

is a critical problem. Actually according to the small inertia of the micro-object, the jump dynamic is not visible with a conventional CCD camera (25 pictures per second). Consequently, this movement can not be controlled. The reduction of this phenomenon is one of major advantages of the liquid medium.

The second important advantage of water is the suppression of the release failures. The experimental statistic classification presented in Table 5 shows that the successful release is higher in water than in air. In case of an automatic micromanipulation station, failures induce the complete stop of the process. The micro-object, which stays attached to the glass microtip must be detached manually with an external tip. An automation of a micromanipulation process needs to eliminate failures.

The normal releases increase from $51 \%$ to $67 \%$ in water but stay a critical problem. Effectively surface forces are not deleted but just reduced in water. Compared to the weight, surface forces stay important in water and could perturb the release tasks.

In conclusion the principal advantage of the water is the significant reduction of jumps and failures. It is a keypoint to perform reliable micromanipulations. The micro-objects release is increased but still remains a critical point in liquid micromanipulation.

\section{Conclusion}

Development of new robotic micro-assembly methods and technologies is a keypoint to fabricate hybrid micro-systems as well as numerous micromechatronic products and requires reliable micromanipulation principles. At present, the release task is the most critical and unreliable phase because of the impact of the surface forces and adhesion forces. A complete modelling of the micro-forces in dry and liquid media 
was presented. These experiments exhibit a correlation better than $40 \%$ between the theoretical forces and the measured forces (except for pull-off in water). This theoretical and experimental comparative analysis between both types of medium shows the potential interest of the liquid in micromanipulation applications. In fact, contact and very large distance force are reduced in liquid while the hydrodynamic force significantly increases. Both phenomena are able to reduce respectively the electrostatic and adhesion perturbations and the loss of micro-objects. Furthermore, experimental pushing and gripping micromanipulations in dry and liquid media confirm the potential interest of liquid. However the release task remains a critical problem. Further works will focus on the study of actuation principles to perform the object release and to obtain a good repeatability of micro-objects positioning notably for automatic micro-assembly tasks.

\section{References}

[1] H. Van Brussel, J. Peirs, D. Reynaerts, A. Delchambre, G. Reinhart, N. Roth, M. Weck, and E. Zussman. Assembly of microsystems. Annals of the CIRP, 49(2):451-472, 2000.

[2] George M. Whitesides and Mila Boncheva. Beyond molecules: Self-assembly of mesoscopic and macroscopic components. Proceedings of the National Academy of Sciences of the United States of America, 99(8):47694774, 2002.

[3] T. Udeshi and K. Tsui. Assembly sequence planning for automated micro assembly. In International Symposium on Assembly and Task Planning, 2005.

[4] N. Dechev, W. L. Cleghorn, and J. K. Mills. Microassembly of 3d microstructures using a compliant, passive microgripper. Journal of Microelectromechanical Systems, 13(2), April 2004.

[5] R. Feynman. Infinitesimal machinery. Journal of Microelectromechanical Systems, 2, No 1:4-14, 1993.

[6] Y. Rollot, S. Regnier, and J-C. Guinot. Micro-robotics : A dynamical model of micro-manipulation by adhesion. In Proc. of the Twelfth CISM-IFToMM Symposium, Theory and Practice of robots and manipulators, Paris, France, July, pages 111-118, 1998.

[7] D.S. Haliyo and S. Régnier. Manipulation of micro-objects using adhesion forces and dynamical effects. In Proceedings of ICRA/IEEE International Conference on Robotics and Automation, May 2002.

[8] R.S. Fearing. A planar milli-robot system on air bearing. In 7th International Symp. Robotics Research, HerrschingGermany, Oct. 1995.

[9] Q. Zhou, B. Chang, and H. N. Koivo. Ambient environment effects in micro/nano handling. In Proc. of the Int. Workshop on Microfactories, pages 146-51, Shangai, China, October 2004.

[10] R. Allen Bowling. A theoretical review of particle adhesion. In Proc. of Symposium on particles on surfaces 1: Detection, Adhesion and Removal, pages 129-142, San Francisco, 1986.

[11] J. Peirs. Design of micromechatronic systems: scale laws, technologies, and medical applications. $\mathrm{PhD}$ thesis, KUL, Belgium, 2001. 
[12] L.-H. Lee. The chemistry and physics of solid adhesion. In L.-H. Lee, editor, Fundamentals of Adhesion. Plenum Press, 1991.

[13] Y. Rollot. Micro-manipulations par adhésion: Modélisations dynamiques et expérimentation. $\mathrm{PhD}$ thesis, Universit Pierre et Marie Curie, Paris, France, 2000.

[14] J. Israelachvili. Intermolecular and Surface Forces. Academic Press, 1991.

[15] Arthur W. Adamson and Alice P. Gast. Physical Chemistry of Surfaces. John Wiley and Sons, $6^{\text {th }}$ edition, 1997.

[16] A. McLachlan. Three-body dispersion forces. Mol. Phys., 7:423-427, 1964.

[17] R. Allen Bowling. A theoretical review of particle adhesion. ParticlesonSurfaces I, 1988.

[18] Z. Xu and R. H. Yoon. The role of hydrophobic interactions in coagulation,. J. Colloid Interface Sci., 44(132):532-541, 1989.

[19] Z. Xu and R.H. Yoon. A study of hydrophobic coagulation. J. Colloid Interface Sci., 45(134):427434, 1990.

[20] X-Y Lin, F. Creuset, and H. Arribart. Atomic force microscopy for local characterzation of surface acid-base properties. J. Phys. Chem., 97:7272-76, 1993.

[21] Nehal I. Abu-Lail and Terri A. Camesano. Role of ionic strength on the relationship of biopolymer conformation, dlvo contributions, and steric interactions to bioadhesion of pseudomonas putida kt2442. Biomacromolecules, 4:1000-12, 2003.

[22] D. S. Rimai and D. J. Quesnel. Fundamentals of Particle Adhesion. Adhesion Society, 2001.

[23] J.A. Greenwood K.L. Johnson. An adhesion map for the contact of elastic spheres. J. Colloid Interface Sci., 192(2):326-333, 1997.

[24] B. V. Derjaguin, V.M. Muller, and YU. P. Toporov. effect of contact deformations on the adhesion of particles. Journal of Colloid and Interface Science, 53(2):314-326, 1975.

[25] Metin Sitti and Hideki Hashimoto. Teleoperated touch feedback from the surfaces at the nanoscale: Modelling and experiments. IEEE-ASME Trans. Mechatron., 8(1):1-12, 2003.

[26] D. Maugis. Adhesion of spheres: the j.k.r-d.m.t transition using a dugdale model. Journal of Colloid and Interface Science, 150(1):243-269, 1992.

[27] Michaël Gauthier, Beatriz Lopez-Walle, and Cédric Clévy. Comparison between micro-objects manipulations in dry and liquid mediums. In proc. of CIRA'05, June 2005.

[28] B. Vgeli and H. von Knel. AFM-study of sticking effects for microparts handling. Wear, 238(1):20$24,2000$.

[29] Q. Ouyang, K. Ishida, and K. Okada. Investigation of micro-adhesion by atomic force microscopy. Applied Surface Science, 169:644-648, 2001.

[30] M. Sitti. Atomic force microcope probe based controlled pushing for nanotribological characterization. IEEE/ASME Transactions on Mechatronics, 9(2):343-349, 2004. 
[31] Pierre-Gilles de Gennes, Françoise Brochart-Wyard, and David Qur. Gouttes, bulles, perles et ondes. Belin, 2002.

[32] J. Agnus, C. Clévy, Y. Urushima, R. Perez, A. Hubert, and N. Chaillet. A micromanipulation station based on a new integrated microprehensile microrobot on chip (mmoc). In Proc. Of the 35th iint. Symposium on Robotics - ISR 2004, Paris, France, March 2004.

[33] C. Clévy, A. Hubert, J. Agnus, and N. Chaillet. A micromanipulation cell including a tool changer. Journal of Micromechanics and Microengineering, 15(1), 2005. 